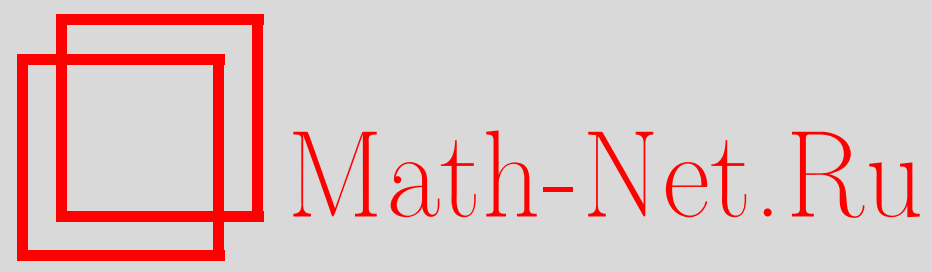

А. Ю. Воловиков, Об одном свойстве функций на сфере, Матем. заметки, 2001, том 70, выпуск 5, 679-690

DOI: https://doi.org/10.4213/mzm780

Использование Общероссийского математического портала Math-Net.Ru подразумевает, что вы прочитали и согласны с пользовательским соглашением http://www.mathnet.ru/rus/agreement

Параметры загрузки:

IP: 35.173 .219 .12

26 апреля 2023 г., 02:42:05

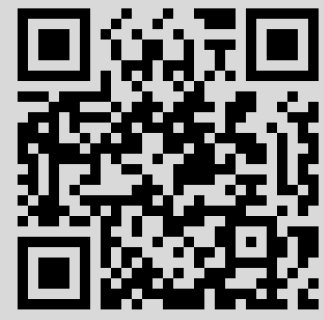




\title{
ОБ ОДНОМ СВОЙСТВЕ ФУНКЦИЙ НА СФЕРЕ
}

\author{
А. Ю. Воловиков
}

Согласно гипотезе Кнастера для любой непрерьвной функции $f: S^{n-1} \rightarrow \mathbb{R}$ и произвольного подмножества из $n$ точек сфферы $S^{n-1}$ найдется вращение, переводящее все эти точки подмножества на линию уровня функции $f$. В статье доказьвается эта гипотеза в случае, когда $n=p^{2}$, где $p$-простое нечетное число, и множество точек лежит на окружности и делит ее на равные части.

Библиографоия: 21 название.

Введение. В работе [1] Кнастер выдвинул гипотезу, что для любого множества точек $v_{1}, \ldots, v_{k} \in S^{k+m-2}$ и произвольного (непрерывного) отображения $f: S^{k+m-2} \rightarrow$ $\mathbb{R}^{m}$ найдется вращение сферы $A \in S O(k+m-1)$ такое, что $f\left(A v_{1}\right)=\cdots=f\left(A v_{k}\right)$.

В настоящее время известно много контрпримеров к этой гипотезе для $m>2$ (см. [2]-[5]). Положительных результатов, связанных с гипотезой Кнастера, не так много. Некоторые из них перечисляются ниже. Обзоры по этой гипотезе см. в [3]-[7].

В этой заметке мы будем рассматривать случай $m=1$. Гипотеза Кнастера в этом случае относится к функциям на сфере $S^{n}$, т.е. к непрерьвньг отображениям $f: S^{n} \rightarrow \mathbb{R}$, и состоит в утверждении, что если заданы функция $f$ и множество точек $v_{1}, \ldots, v_{n+1} \in$ $S^{n}$, то найдется вращение $A \in S O(n+1)$ (зависящее от множества точек и функции) такое, что $f\left(A v_{1}\right)=\cdots=f\left(A v_{n+1}\right)$. Иньми словами, требуется доказать, что для любой непрерьвной функции, определенной на сфере $S^{n}$, и любого множества из $n+1$ точек на сфере найдется вращение, переводящее эти $n+1$ точек на поверхность уровня функции.

Из результатов Флойда [8], показавшего, что любые три точки на двумерной сфере можно вращением поместить на линию уровня любой заданной (непрерывной) функции $f$, вытекает, что гипотеза Кнастера верна для $n=2$.

Классическим результатом в случае $n>2$ является результат Ямабэ-Юдзёбо [9], доказанньй другим способом Янгом [10] и утверждаюший, что множество из $n+1$ точек на $n$-мерной сфере, радиус векторы которых образуют ортонормированньй базис в $\mathbb{R}^{n+1}$, удовлетворяет гипотезе Кнастера.

В случае $n>2$ все множества, для которых доказана справедливость гипотезы Кнастера, обладают той или иной степенью симметрии. Обзор результатов для множеств,

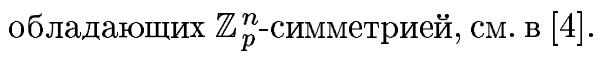

Работа выполнена при финансовой поддержке Российского фонда фундаментальных исследований, грант № 00-01-00304, и фонда INTAS, грант № 96-0712. 
В настоящей заметке, основьваясь на имеющих самостоятельньй интерес усилениях результатов Манкхольма [11], мы покажем, что гипотеза Кнастера справедлива для множества из $p^{2}$ точек, лежащих на окружности в вершинах правильного $p^{2}$-угольника, где $p$ - нечетное простое число, а также для множества из $p^{2}+1$ точек, которое получается из описанного вьше добавлением одной точки - любого конца диаметра, перпендикулярного плоскости окружности. При этом размерность сферы, на которой определена функция, меньше, чем $p^{2}-1$. Группой симметрии в этом случае будет служить циклическая группа порядка $p^{2}$. Аналогичные результаты для групшы симметрии $\mathbb{Z}_{p}$ рассматривались в [7], [12]-[14].

Некоторые результаты настоящей заметки анонсированы в [13] и [14].

1. G-отображения сферы в многообразие 2-реперов. Обозначим через $V_{n}$ многообразие Штифеля $V_{n, 2}$ ортонормированных 2 -реперов в $\mathbb{R}^{n}$. На $V_{n}$ действует окружность $S^{1}$ следующим образом. Элемент $e^{i \varphi} \in S^{1}$ действует на $(u, v) \in V_{n}$ поворотом этого репера на угол $\varphi$ в плоскости векторов $u, v($ от $u$ к $v$ ). Если отождествить плоскость, натянутую на векторы $u, v$, с комплексными числами $\mathbb{C}$, переведя $u$ в 1 и $v$ в $i \in \mathbb{C}$, то $e^{i \varphi}$ будет действовать умножением на $e^{i \varphi}$. Это действие является свободньм. Таким образом, любая циклическая группа (как подгруппа $S^{1}$ ) действует свободно на $V_{n}$.

На единичной сфере $S^{2 n-1} \subset \mathbb{C}^{n}$ окружность свободно действует также умножением на $e^{i \varphi}$.

Пусть $G$ - циклическая группа нечетного порядка. Тогда $G$ свободно действует на $S^{2 n-3}$ и $V_{n}$. Поскольку размерность многообразия $V_{n}$ равна $2 n-3$, имеется эквивариантное отображение $V_{n} \rightarrow S^{2 n-3}$ (в силу $(2 n-3)$-универсальности сферы $S^{2 n-3}$ ).

Лемма 1. Для нечетного $n$ имеется $G$-отображсение $S^{2 n-3} \rightarrow V_{n}$.

ДоказАтЕльство. Обозначим через $E_{G} \rightarrow B_{G}$ универсальное главное $G$-расслоение. Можно считать, что $E_{G}=S^{\infty}$ - бесконечномерная сфера и что $(2 k-1)$-мерный остов $E_{G}$ есть сфера $S^{2 k-1}$ с указанным выше действием $G$. Как известно, $G$-отображения $S^{2 n-3} \rightarrow V_{n}$ взаимно однозначно соответствуют сечениям расслоения $S^{2 n-3} \times{ }_{G} V_{n}$ со слоем $V_{n}$, ассоциированного с главным расслоением $S^{2 n-3} \rightarrow S^{2 n-3} / G$. Чтобы построить такое сечение, достаточно построить сечение расслоения $E_{G} \times_{G} V_{n} \rightarrow B_{G}$ над $(2 n-3)$-мерным остовом. Фундаментальная группа $\pi_{1}\left(B_{G}\right)=G$ действует на гомотопических группах многообразия $V_{n}$ тривиальным образом (поскольку $G$ действует как подгруппа окружности). Поэтому препятствия к построению сечения лежат в групах $H^{i}\left(G ; \pi_{i-1}\left(V_{n}\right)\right)$ (с постоянной системой коэффициентов). Если $n$ нечетно, то $V_{n}$ является когомологической $(2 n-3)$-мерной сферой по модулю $p$ для любого простого нечетного числа $p$ (см. [15]) и, следовательно, гомотопические групшы $\pi_{j}\left(V_{n}\right)$ многообразия $V_{n}$ являются 2-группами в размерностях $0<j<2 n-3$ (см. [15, гл. $5, \S 6])$. Поскольку порядок $G$ - нечетное число, указанные выше группы когомологий тривиальны при $i \leqslant 2 n-3$ и, следовательно, можно построить искомое сечение над $(2 n-3)$-мерным остовом.

ЗАмЕчаниЕ 1 . Нетрудно видеть, что при четном $n$ не существует $G$-отображения $S^{2 n-3} \rightarrow V_{n}$. Действительно, предполагая противное, рассмотрим композицию этого отображения с $G$-отображением $V_{n} \rightarrow S^{2 n-3}$. Тогда полученное $G$-отображение $S^{2 n-3} \rightarrow S^{2 n-3}$ должно иметь ненулевую степень. С другой стороны, $V_{n}$ имеет такие 
же целочисленные когомологии, что и произведение сфер $S^{n-2} \times S^{n-1}$ (см. [16, предложение 10.1]. Поэтому отображение $S^{2 n-3} \rightarrow V_{n}$ индуцирует тривиальный гомоморфизм в когомологиях, что приводит к противоречию.

Поскольку $V_{n-1} \subset V_{n}$, при четном $n$ имеется $G$-отображение $S^{2 n-5} \rightarrow V_{n}$.

Далее нам будет удобно представлять действие циклической группы $G=\mathbb{Z}_{q}$ на $V_{n}=$ $V_{n, 2}$ следующим образом. Пусть $T$ - образующая групшы $G=\mathbb{Z}_{q}$ и $(u, v) \in V_{n}$. Положим $\left(u_{i+1}, v_{i+1}\right)=T^{i}(u, v)$, где $i=0, \ldots, q-1$. Тогда точка $(u, v)$ однозначно соответствует $q$-набору точек $\left(u_{1}, \ldots, u_{q}\right)$, которые являются вершинами правильного $q$-угольника, и $G$ действует циклическими перестановками. Таким образом, $V_{n}$ эквивариантно гомеоморфно подпространству в произведении $S^{n-1} \times \cdots \times S^{n-1}$, состоящему из точек вида $\left(C u_{1}^{0}, \ldots, C u_{q}^{0}\right)$, где $C \in S O(n)$ и $\left(u_{1}^{0}, \ldots, u_{q}^{0}\right)$ - один из наборов указанного выше вида.

Будем рассматривать $S^{n-1}$ как экватор в $S^{n}$. Пусть $e-$ северньй полюс. Если $\left(z_{1}, \ldots, z_{q}\right) \in V_{n}=V_{n, 2}$, то набор $\left(z_{1}, \ldots, z_{q}, e\right)$ можно рассматривать как точку пространства $V_{n+1,3}$. Пусть $A_{t} \in S O(n+1), t \in I=[0,1],-$ такая непрерьвная кривая, что кривая $A_{t}$ е вложена в $S^{n}$. Тогда, переводя $\left(z_{1}, \ldots, z_{q}\right) \in V_{n, 2}$ в $\left(A_{t} z_{1}, \ldots, A_{t} z_{q}, A_{t} e\right)$, получаем вложение $V_{n, 2} \times I \subset V_{n+1,3}$. Пусть $G=\mathbb{Z}_{q}$ действует на $V_{n+1,3}$ циклическими перестановками первых $q$ точек в наборе, а на $V_{n, 2} \times I$ действует, как на произведении $\mathbb{Z}_{q}$-пространств, причем на $I$ действие тривиально. Легко видеть, что указанное вьше вложение является эквивариантньм отображением.

2. Теоремы типа Борсука-Улама для пространств с действием группы $\mathbb{Z}_{p^{\alpha}}$. Пусть $X$ - свободное $G$-пространство, где $G$ - конечная группа, и $f: X \rightarrow Y-$ непрерьвное отображение в пространство $Y$. Положим

$$
A(f)=\{x \in X \mid f(x)=f(g x) \forall g \in G\} .
$$

Скажем, что $A(f) C$-существенно, если для любой гомотопии $F: X \times I \rightarrow Y$ отображения $f$ в $A(F) \subset X \times I$ имеется компонента, соединяющая основания цилиндра (здесь $I$ - отрезок $[0,1]$ с тривиальным действием групшы $G)$.

Далее в этом пункте мы будем считать, что $G=\mathbb{Z}_{p^{\alpha}}$, где $p$ - нечетное простое число.

Рассмотрим нечетномерную сферу $S^{2 n-3}$ со стандартным свободным действием группы $G$ (см. выше). Пусть $f: S^{2 n-3} \rightarrow \mathbb{R}^{m}$ - непрерьвное отображение. Естественно было бы предполагать, что $A(f)$ имеет размерность, не меньшую, чем $2 n-3-m(|G|-1)$, поскольку $A(f)$ задается системой из $m(|G|-1)$ уравнений. А. С. Шварц показал, что при $\alpha=1$ это действительно так [17]. Манкхольм [11] обнаружил, что для $\alpha>1$ это уже неверно, и нашел следующую оценку (которая, как он показал в той же работе, не является точной):

$$
\begin{aligned}
\operatorname{dim} A(f) & \geqslant 2 n-3-\left(p^{\alpha}-1\right) m-\left[m(\alpha-1) p^{\alpha}-(m \alpha+2) p^{\alpha-1}+m+3\right] \\
& =2 n-6-\alpha m p^{\alpha}+(m \alpha+2) p^{\alpha-1} .
\end{aligned}
$$

Эта же оценка справедлива и для любого свободного действия групшы $G$ на $S^{2 n-3}$.

Основьваясь на рассуждениях из работы [11], мы докажем более общее утверждение, следствие из которого нам понадобится в следующем пункте.

Пусть $X$-компактноеметрическое свободное $G$-пространство и $f: X \rightarrow \mathbb{R}^{m}$-непрерьвное отображение. 
ТЕорема 1. Предположим, что существует $G$-отображсние $S^{2 n-3} \rightarrow X u$ $2 n-6-\alpha m p^{\alpha}+(m \alpha+2) p^{\alpha-1} \geqslant 0$. Тогда $A(f)$ является $C$-существенным и

$$
\operatorname{dim} A(f) \geqslant 2 n-6-\alpha m p^{\alpha}+(m \alpha+2) p^{\alpha-1} .
$$

Если $F: X \times I \rightarrow \mathbb{R}^{m}$ - гомотопия отображения $f$ и $h: X \times I \rightarrow \mathbb{R}$ - непрерывная инвариантная относительно действия $G$ функция, неотрицательная на верхнем основании и неположительная на нижнем основании, то

$$
\operatorname{dim} A(F) \cap h^{-1}(0) \geqslant 2 n-6-\alpha m p^{\alpha}+(m \alpha+2) p^{\alpha-1} .
$$

Для доказательства нам понадобится следующая лемма, в которой через $e(\zeta)$ обозначается эйлеров класс в комплексной $K$-теории комплексного векторного расслоения $\zeta$.

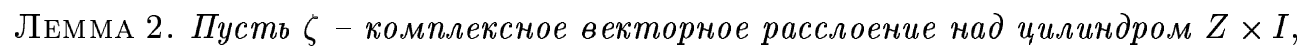
әде $Z$ - компакт и $I=[0,1]$, а $s$ - некоторое его сечение над $Z \times I$. Пусть $A-$ множсество нулей сечения $s$. Тогда если $e(\zeta) \neq 0$, то в $A$ имеется компонента, соединяющая основания иилиндра.

Предположим дополнительно, что имеется комплексное векторное расслоение $\zeta^{\prime}$ над $Z \times I$ комплексной размерности $d$ такое, что е $\left(\zeta \oplus \zeta^{\prime}\right) \neq 0$. Тогда если $h: Z \times I \rightarrow \mathbb{R}$ - непрерывная функция, неотрицательная на верхнем основании $u$ неположительная на нижнем основании, то $\operatorname{dim} A \cap h^{-1}(0) \geqslant 2 d$.

ДокАЗАТЕЛЬСтво. Положим $A_{t}=A \cap Z \times\{t\}$, где $t \in[0,1]$. Поскольку $e(\zeta) \neq 0$ и $K(Z \times I)=K(Z)$, имеем $A_{t} \neq \varnothing$ при любом $t$.

Доказательство проведем от противного. Тогда найдутся открытые множества $U_{0}, U_{1}$ такие, что

$$
A \subset U_{0} \cup U_{1}, \quad U_{0} \cap U_{1}=\varnothing, \quad Z \times\{0\} \subset U_{0}, \quad Z \times\{1\} \subset U_{1} .
$$

В частности, $U_{0} \cap Z \times\{1\}=\varnothing$ и $U_{1} \cap Z \times\{0\}=\varnothing$. Положим $N_{0}=Z \times I \backslash U_{0}$, $N_{1}=Z \times I \backslash U_{1}$. Тогда $N_{0} \cap N_{1} \subset Z \times I \backslash A$ и $Z \times\{0\} \subset N_{1}, Z \times\{1\} \subset N_{0}$.

Обозначим через $e_{0}$ образ $e(\zeta)$ в $K\left(N_{0}\right)$. Рассмотрим отрезок последовательности Майера-Виеториса

$$
K(Z \times I) \rightarrow K\left(N_{0}\right) \oplus K\left(N_{1}\right) \rightarrow K\left(N_{0} \cap N_{1}\right)
$$

Элемент $e_{0} \oplus 0 \in K\left(N_{0}\right) \oplus K\left(N_{1}\right)$ переходит в нуль в группе $K\left(N_{0} \cap N_{1}\right)$. Действительно, образ этого элемента является эйлеровым классом векторного расслоения $\left.\zeta\right|_{N_{0} \cap N_{1}}$, которое имеет ненулевое сечение (и, следовательно, его эйлеров класс тривиален), поскольку пересечение $A$ с $N_{0} \cap N_{1}$ пусто. Из точности последовательности Майера-Виеториса следует, что существует $\alpha \in K(Z \times I)$ такой, что его образ в $K\left(N_{0}\right)$ равен $e_{0}$, а образ в $K\left(N_{1}\right)$ равен нулю. Поскольку $K(Z \times I)=K(Z)$ и $Z \times\{0\} \subset N_{1}$, мы получаем, что $\alpha=0$. Аналогично, пользуясь тем, что $Z \times\{1\} \subset N_{0}$, и тем, что $e_{0}$ является образом как класса $\alpha$, так и класса $e(\zeta) \neq 0$, получаем, что $\alpha \neq 0$. Полученное противоречие доказывает первое утверждение леммы.

Для доказательства второго утверждения положим $N_{0}=\{h \geqslant 0\}, N_{1}=\{h \leqslant 0\}$. Тогда $Z \times\{1\} \subset N_{0}, Z \times\{0\} \subset N_{1}$ и $N_{0} \cap N_{1}=h^{-1}(0)$. Рассуждения, приведенные 
выше, показывают, что эйлеров класс ограничения расслоения $\zeta \oplus \zeta^{\prime}$ на $h^{-1}(0)$ не равен нулю.

Положим $A_{0}=A \cap h^{-1}(0)$. Расслоение $\left.\zeta^{\prime}\right|_{A_{0}}$ не может иметь нигде ненулевого сечения. Действительно, если бы такое сечение существовало, его можно было продолжить до сечения $s^{\prime}$ расслоения $\left.\zeta^{\prime}\right|_{h^{-1}(0)}$. Тогда $s \oplus s^{\prime}$ - нигде ненулевое сечение расслоения $\left.\left(\zeta \oplus \zeta^{\prime}\right)\right|_{h^{-1}(0)}$ и, следовательно, эйлеров класс этого расслоения равен нулю. Полученное противоречие доказьвает сделанное утверждение.

Для завершения доказательства леммы достаточно установить следующий факт (частньй случай которого доказан в [11]): векторное расслоение $\mu$ (комплексное или вещественное) вещественной размерности $k$ над компактом $Y c \operatorname{dim} Y<k$ имеет всюду ненулевое сечение.

Расслоение $\mu$ индуцировано из тавтологического расслоения над грассмановым многообразием $G_{N, k}$, где $N$ достаточно велико, некоторым отображением $Y \rightarrow G_{N, k}$. Заменив, если необходимо, это отображение гомотопным отображением, можно считать, что оно пропускается через нерв $K$ достаточно мелкого покрытия пространства $Y$. Поскольку $\operatorname{dim} Y<k$, можно считать, что $\operatorname{dim} K<k$. Таким образом, $\mu$ изоморфно векторному расслоению, индуцированному из $k$-мерного векторного расслоения над $C W$-комплексом размерности $<k$. Из теории препятствий следует, что последнее расслоение имеет нигде ненулевое сечение, поэтому и $\mu$ имеет такое сечение.

ДОКАЗАТЕЛЬСТВО ТЕОРЕМЫ 1 . Пусть $Y$ - свободное $G$-пространство и $L$ - пространство комплексного представления групшы $G$. Тогда $Y \times_{G} L \rightarrow Y / G-$ комплексное векторное расслоение над $Y / G$.

Обозначим через $\mathbb{C}$ представление $G$, при котором образующий $G$ действует умножением на $e^{2 \pi i / q}, q=p^{\alpha}$. Через $d \mathbb{C}$ обозначим прямую сумму $d$ экземпляров представления $\mathbb{C}$, где

$$
d=n-3+p^{\alpha-1}-\frac{1}{2} \alpha m\left(p^{\alpha}-p^{\alpha-1}\right) .
$$

Обозначим через $\xi \oplus \xi^{\prime}$ расслоение $S^{2 n-3} \times_{G}(W \oplus d \mathbb{C}) \rightarrow S^{2 n-3} / G$, где $W=\left(\mathbb{R}^{m} \times \cdots\right.$ $\left.\times \mathbb{R}^{m}\right) / \Delta, \Delta$ - диагональ и в произведении взято $|G|=p^{\alpha}$ сомножителей. Таким образом, $\operatorname{dim}_{\mathbb{R}} W=m\left(p^{\alpha}-1\right)$. Образующий группы $G$ действует на $W$ как циклическая перестановка. Поскольку $G$ оставляет неподвижным только нулевой вектор, представление $W$ вещественно изоморфно комплексному представлению. Манкхольм показал в [11] (см. также [18]), что $e\left(\xi \oplus \xi^{\prime}\right) \neq 0$ в $\widetilde{K}\left(S^{2 n-3} / G\right)$.

Поскольку имеется эквивариантное отображение $S^{2 n-3} \rightarrow X$, мы получаем, что $\xi \oplus \xi^{\prime}$ индуцировано из расслоения $X \times_{G}(W \oplus d \mathbb{C}) \rightarrow X / G$. Поэтому отличен от нуля эйлеров класс в комплексной $K$-теории векторного расслоения $(X \times I) \times_{G}(W \oplus d \mathbb{C}) \rightarrow Z \times I$, где $Z=X / G$, которое мы обозначим через $\zeta \oplus \zeta^{\prime}$.

В [11] показано, что по $F: X \times I \rightarrow \mathbb{R}^{m}$ естественным образом строится сечение $s=s_{F}$ расслоения $\zeta$, множество нулей которого совпадает с $A=A(F) / G$.

Для построения такого сечения определим отображение $\widehat{F}: X \times I \rightarrow W$ формулой

$$
\widehat{F}(x, t)=\left(F(x, t), F(T x, t), \ldots, F\left(T^{q} x, t\right)\right),
$$

где $T$ - образуюший группы $G$. Легко видеть, что $\widehat{F}$ эквивариантно, поэтому отображение $X \times I \rightarrow(X \times I) \times W$, переводящее $(x, t)$ в $(x, \widehat{F}(x, t))$, также является $G$-отображени- 
ем. Отображение факторпространств, индуцированное последним $G$-отображением, и есть требуемое сечение $s$.

Теперь мы находимся в условиях леммы 2 , где вместо $h$ нужно, пользуясь инвариантностью этой функции, взять возникающую функцию $\bar{h}: X / G \times I \rightarrow \mathbb{R}$. Поскольку размерности пространств $A(F) \cap h^{-1}(0)$ и $A(F) / G \cap \bar{h}^{-1}(0)$ одинаковы, применяя лемму 2 , получаем, что $A(f) C$-существенно и

$$
\begin{aligned}
\operatorname{dim} A(F) \cap h^{-1}(0) & \geqslant 2 \operatorname{dim}_{\mathbb{C}} \zeta^{\prime}=2 n-6+2 p^{\alpha-1}-\alpha m\left(p^{\alpha}-p^{\alpha-1}\right) \\
& =2 n-6+p^{\alpha-1}(\alpha m+2)-\alpha m p^{\alpha} .
\end{aligned}
$$

Оценка размерности $A(f)$ вытекает из предыдущей оценки, если определить $F$ и $h$ формулами

$$
F(x, t)=f(x), \quad h(x, t)=t-1, \quad x \in X, \quad t \in[0,1] .
$$

Тогда $h^{-1}(0)=X \times\{1\}$ и после естественного отождествления последнего пространства с $X$ имеем $A(F) \cap h^{-1}(0)=A(f)$. Доказательство теоремы 1 закончено.

ЗАмЕчаниЕ 2. $C$-существенность множества $A(f)$ можно вьвести из общего результата Александера-Йорка [19] (см. также статью Аллигуда [20]), используя тот факт, что $e(\zeta) \neq 0$.

СледСТВИЕ 1. Пусть $G=\mathbb{Z}_{p^{2}}$, әде $p$ - нечетное простое число, и $f: V_{n} \rightarrow \mathbb{R}-$ непрерывная функиия. Тогда $A(f)$ непусто и $C$-существенно, если $n \geqslant p^{2}-2 p+4$.

ДокаЗАТЕЛьство. Достаточно проверить утверждение для $n=p^{2}-2 p+4$. Так как $n$ нечетно, из леммы 1 следует, что имеется $G$-отображение $S^{2 n-3} \rightarrow V_{n}$. Из теоремы 1 вытекает, что $A(f)$ является $C$-существенным и $\operatorname{dim} A(f) \geqslant 2 n-6-2 p^{2}+4 p=2$; следовательно, $A(f) \neq \varnothing$.

ЗАмЕчАниЕ 3 . Манкхольм показал в [11], что для $G=\mathbb{Z}_{25}$ и любого $h: S^{31} \rightarrow \mathbb{R}$ множество $A(h)$ непусто. Поэтому из леммы 1 вытекает, что $A(f) \neq \varnothing$ для любого $f: V_{17} \rightarrow \mathbb{R}$.

ЗАмЕчАниЕ 4 . Для действия групшы $G=\mathbb{Z}_{27}$ на $V_{21}$ имеет место следуюший результат: для любого $f: V_{21} \rightarrow \mathbb{R}$ множество $A(f)$ непусто и $C$-существенно. Это непосредственно вытекает из леммы 1 и теоремы 1 (по теореме Манкхольма для $G=\mathbb{Z}_{27}$ и любого $h: S^{39} \rightarrow \mathbb{R}$ множество $A(h)$ непусто).

Приведем теперь усиление "обратной теоремы Борсука-Улама" для групшы $\mathbb{Z}_{p^{\alpha}}$, доказанной Манкхольмом в [11]. Наши рассуждения основаны на тех же идеях.

Далее мы предполагаем, что $G=\mathbb{Z}_{p^{\alpha}}$, где $p$ - нечетное простое число и $\alpha>1$.

Манкхольм показал в [11], что если $2 n-3 \leqslant m\left(p^{\alpha}-1\right)+(2 p-3) m-1$ и $p^{\alpha} \neq 9$, то существует $f: S^{2 n-3} \rightarrow \mathbb{R}^{m}$ с $A(f)=\varnothing$.

Лемма 3. Пусть $\eta_{1}, \ldots, \eta_{r}$ - векторные расслоения над конечным или счетным $C W$-комплексом $X$. Предположим, что при любом $i=1, \ldots, r$ расслоение $\eta_{i}$ имеет ненулевое сечение над $m_{i}$-мерным остовом комплекса $X$. Тогда $\eta_{1} \oplus \cdots \oplus \eta_{r}$ имеет ненулевое сечение над l-мерным остовом пространства $X$, əде $l=r-1+\sum_{i=1}^{r} m_{i}$. 
ДоказАтЕЛьство. Обозначим $k$-мерньй остов $C W$-комплекса $X$ через $X_{k}$. Пусть $s_{1}, \ldots, s_{r}$ - сечения над $X$ расслоений $\eta_{1}, \ldots, \eta_{r}$ такие, что $s_{i}$ не обращается в нуль ни в одной точке $m_{i}$-мерного остова $X_{m_{i}}$. Тогда $s_{1} \times \cdots \times s_{r}$ является сечением расслоения $\eta_{1} \times \cdots \times \eta_{r}$ с базой $X^{r}$, ненулевьп над объединением подпространств вида $X \times \cdots \times X_{m_{i}} \times \cdots \times X$, где $i$-й сомножитель равен $X_{m_{i}}$ и остальные $r-1$ сомножителей равны $X$. Пусть $\Delta: X \rightarrow X^{r}$ - какая-нибудь клеточная аппроксимация диагонали $d X=\left\{(x, \ldots, x) \in X^{r} \mid x \in X\right\} \subset X^{r}$. Тогда $\eta_{1} \oplus \cdots \oplus \eta_{r}=\Delta^{*}\left(\eta_{1} \times \cdots \times \eta_{r}\right)$ и $\Delta^{*}\left(s_{1} \times \cdots \times s_{r}\right)$ - сечение расслоения $\eta_{1} \oplus \cdots \oplus \eta_{r}$, являющееся ненулевьм над $X_{l}$. Действительно, $\Delta\left(X_{l}\right)$ содержится в $l$-мерном остове пространства $X^{r}$, который является объединением подпространств вида $X_{k_{1}} \times \cdots \times X_{k_{r}}$, где $\sum_{i=1}^{r} k_{i} \leqslant l$. Пусть $\Delta(x)=\left(x_{1}, \ldots, x_{r}\right) \in X_{k_{1}} \times \cdots \times X_{k_{r}}$, где $x \in X_{l}$. Из неравенства $\sum_{i=1}^{r} k_{i} \leqslant l$ следует, что $k_{i} \leqslant m_{i}$ при некотором $i$ (в противном случае $\sum_{i=1}^{r} k_{i} \geqslant l+1$ ). Следовательно, $s_{i}\left(x_{i}\right) \neq 0$, что влечет $\Delta^{*}\left(s_{1} \times \cdots \times s_{r}\right)(x) \neq 0$.

Рассмотрим все положительные целые числа, делящиеся на $p$ и не превосходящие $\left(p^{\alpha}-1\right) / 2$. Обозначим через $Q\left(p^{\alpha}, m\right)$ множество с повторением, составленное из чисел указанного вида, в котором каждое число повторено $m$ раз. Обозначим через $L\left(p^{\alpha}, m\right)$ наибольшее из чисел $r$ таких, что существует разбиение множества $Q\left(p^{\alpha}, m\right)$ на $r$ непересекающихся подмножеств таких, что произведение всех чисел из каждого подмножества делится на $p^{\alpha}$.

Пользуясь тем, что среди целых положительных чисел, не превосходящих $\left(p^{\alpha}-1\right) / 2$, имеется $\left(p^{\alpha-j}-1\right) / 2$ чисел, делящихся на $p^{\alpha-j}, j=1, \ldots, \alpha-1$, можно показать, что $\left(\left(p^{\alpha}-1\right) / 2\right) !^{m}$ делится на $p^{A}$ и не делится на $p^{A+1}$, где $A=m\left(p^{\alpha}-\alpha p+\alpha-1\right) /(2(p-1))$. Поэтому

$$
L\left(p^{\alpha}, m\right) \leqslant\left[\frac{m\left(p^{\alpha}-\alpha p+\alpha-1\right)}{2 \alpha(p-1)}\right],
$$

где квадратными скобками обозначена целая часть числа. Для небольших $\alpha$, например для $\alpha \leqslant 8$, нетрудно непосредственно проверить, что имеет место равенство (индукцией нетрудно доказать, что имеет место равенство в случае, когда $\alpha$ является степенью двойки). В частности, в случае $\alpha=2$, представляющем основной интерес в этой заметке, имеем $L\left(p^{2}, m\right)=[m(p-1) / 4]$.

Tеорема 2. Если $2 n-3 \leqslant m\left(p^{\alpha}-1\right)+(2 p-3) L\left(p^{\alpha}, m\right)-1$, mo cyществует отобрахсение $f: S^{2 n-3} \rightarrow \mathbb{R}^{m}$ такое, что $A(f)=\varnothing$.

ДоказАТЕЛьСтво. Обозначим через $\lambda$ линейное расслоение $E_{G} \times_{G} \mathbb{C} \rightarrow B_{G}$, где, как и вьше, на $\mathbb{C}$ образующий грушшы $G$ действует как умножение на $e^{2 \pi i / p^{\alpha}}$. Можно считать, что $E_{G}=S^{\infty} \subset \mathbb{C}^{\infty}$. Положим $\xi=\lambda \oplus \lambda^{2} \oplus \cdots \oplus \lambda^{k}$, где $k=\left(p^{\alpha}-1\right) / 2$ и $\lambda^{j}$ есть $j$-я тензорная степень расслоения $\lambda$. В [11] показано, что ненулевое сечение расслоения $m \xi=\xi \oplus \cdots \oplus \xi$ над $S^{2 N-3} / G$ дает $f: S^{2 N-3} \rightarrow \mathbb{R}^{m}$ с $A(f)=\varnothing$ (отметим, что $m \xi$ изоморфно расслоению $E_{G} \times_{G} W \rightarrow B_{G}$ и $S^{2 N-3} / G$ можно считать $(2 N-3)$-мерным остовом пространства $\left.B_{G}\right)$.

Хорошо известно, что $H^{*}\left(B_{G} ; \mathbb{Z}\right)=\mathbb{Z}_{p^{\alpha}}[u]$, где $\operatorname{deg} u=2$.

Имеем

$$
e\left(\lambda^{i_{1}} \oplus \cdots \oplus \lambda^{i_{s}}\right)=e\left(\lambda^{i_{1}}\right) \cdots e\left(\lambda^{i_{s}}\right)=c_{1}\left(\lambda^{i_{1}}\right) \cdots c_{1}\left(\lambda^{i_{s}}\right)=i_{1} \cdots i_{s} u^{s}
$$


где $e$ и $c_{1}-$ классы Эйлера и Чженя соответственно. Если $i_{1} \cdots i_{s}$ делится на $p^{\alpha}$, то указанный класс тривиален (заметим, что $\left.e(m \xi)=(k !)^{m} u^{k m}\right)$.

Положим $r=L\left(p^{\alpha}, m\right)$; тогда $m \xi$ можно представить в виде $m \xi=\eta_{1} \oplus \cdots \oplus \eta_{r}$, где $e\left(\eta_{i}\right)=0, i=1, \ldots, r$. (Манкхольм в [11] использовал разложение $m \xi=\xi \oplus \cdots \oplus \xi$.) Пусть $\operatorname{dim}_{\mathbb{R}} \eta_{i}=2 m_{i} ;$ тогда $2 \sum_{i=1}^{r} m_{i}=m\left(p^{\alpha}-1\right)$.

Поскольку $е\left(\eta_{i}\right)=0$, расслоение $\eta_{i}$ имеет ненулевое сечение над $2 m_{i \text {-мерным остовом }}$ пространства $B_{G}$. Дальнейшие препятствия лежат в группах когомологий

$$
H^{2 m_{i}+t}\left(B_{G} ; \pi_{2 m_{i}-1+t}\left(S^{2 m_{i}-1}\right)\right)
$$

Системы коэффициентов постоянны в силу того, что любое комплексноевекторное расслоение ориентируемо. Указанные группы тривиальны при $0<t<2 p-3$, поскольку группы $\pi_{2 m_{i}-1+t}\left(S^{2 m_{i}-1}\right)$ при таких $t$ не имеют $p$-кручения [15]. Таким образом, имеется ненулевое сечение расслоения $\eta_{i}$ над $\left(2 m_{i}+2 p-4\right)$-мерньп остовом пространства $B_{G}$. Из леммы 3 следует, что расслоение $m \xi$ имеет ненулевое сечение над $l$-мерным остовом пространства $B_{G}=S^{\infty} / G$, где

$$
l=-1+\sum_{i=1}^{r}\left(2 m_{i}+2 p-3\right)=m\left(p^{\alpha}-1\right)+(2 p-3) r-1,
$$

что и требовалось доказать.

В заключение отметим, что вместо $S^{2 n-3}$ в теореме 2 можно взять любое компактное свободное $G$-пространство $Y$ с $\operatorname{dim} Y \leqslant 2 n-3$, поскольку существует $G$-отображение такого $Y$ в $S^{2 n-3}$. В частности, это верно для $Y=V_{n}$.

3. Приложения к гипотезе Кнастера. В этом пункте мы применим результаты п. 2 к гипотезе Кнастера. Через $G$ будет обозначаться циклическая группа $\mathbb{Z}_{p^{2}}$ порядка $p^{2}$, где $p$ - простое нечетное число.

Пусть $S^{N}$ - единичная сфера в $\mathbb{R}^{M}, M>N$, задающаяся уравнениями

$$
x_{1}^{2}+\cdots+x_{N+1}^{2}=1
$$

и $x_{i}=0$ при $i>N+1$. Предположим, что $R \subset S^{N}$ - конечное множество точек. Для $A \in S O(N+1)$ обозначим через $A R$ множество точек, полученных из $R$ с помошью вращения $A$. Обозначим через $l$ размерность линейной оболочки радиус-векторов точек из $R$. Тогда можно найти вращение $B \in S O(N+1)$ такое, что $B R \subset S^{l-1} \subset S^{m}$, где $m \geqslant l-1$. Обозначим через $k(R)$ наименьшее из чисел $m$, для которых выполнено следующее свойство: для любой функции $f: S^{m} \rightarrow \mathbb{R}$ найдется вращение $C \in S O(m+1)$, переводящее $B R$ в поверхность уровня функции $f$. Из определения ясно, что $k(R)=k(A R)$ для произвольного вращения $A \in S O(N+1)$. Гипотеза Кнастера состоит в том, что $k(R) \leqslant|R|-1$, где $|R|$ - количество точек в $R$.

Пусть $S^{N}$ - сфера единичного радиуса в $\mathbb{R}^{N+1}$ и $S^{2} \subset S^{N}$ - единичная двумерная сфера. Для $0 \leqslant \rho<\infty$ определим множество $R_{\rho} \subset S^{2}$ такое, что $\left|R_{0}\right|=p^{2}$ и $\left|R_{\rho}\right|=p^{2}+1$ при $\rho>0$, следующим образом: $R_{0}$ состоит из $p^{2}$ вершин правильного $p^{2}$-угольника, вписанного в экватор сферы $S^{2}$. 
Для того чтобы определить $R_{\rho}$ при $\rho>0$, рассмотрим окружность, которая получается в результате сечения сферы $S^{2}$ некоторой плоскостью, параллельной экваториальной плоскости (или совпадающей с последней). По определению множество $R_{\rho}$ при $\rho>0$ состоит из $p^{2}$ вершин правильного $p^{2}$-угольника, вписанного в эту окружность, и северного полюса сферы $S^{2}$. Число $0<\rho<\infty$ определяется как отношение расстояния от северного полюса до центра $p^{2}$-угольника к радиусу окружности, в которую вписан многоугольник. Ясно также, что множество $R_{\rho_{1}}$ не может быть получено из $R_{\rho_{2}}$ врашением сферы при $\rho_{1} \neq \rho_{2}$.

Теорема 3. Выполнено $k\left(R_{0}\right) \leqslant p^{2}-2 p+3 u k\left(R_{\rho}\right) \leqslant p^{2}-2 p+4$ при $\rho>0$.

ДокАЗАТЕльство. Утверждение относительномножества $k\left(R_{0}\right)$ вытекает непосредственно из следствия 1. Действительно, пусть $f: S^{p^{2}-2 p+3} \rightarrow \mathbb{R}$ - непрерьвная функция. Определим $g: V_{p^{2}-2 p+4} \rightarrow \mathbb{R}$ как $g(z)=f\left(v_{1}\right)$, где $z=\left(v_{1}, v_{2}\right) \in V_{p^{2}-2 p+4}$. Таким образом, $g=f \circ \nu_{1}$, где $\nu_{1}: V_{p^{2}-2 p+4} \rightarrow S^{p^{2}-2 p+3}$ - проекция на первьй вектор. Из следствия 1 вытекает, что $A(g) \neq \varnothing$; поэтому имеется орбита, на которой $g$ постоянна. Легко видеть, что проекция этой орбиты при отображении $\nu_{1}$ есть множество точек, конгруэнтное $R_{0} \subset S^{p^{2}-2 p+3}$, и функция $f$ постоянна на этом множестве.

Утверждение относительно множества $R_{\rho}$ при $\rho>0$ доказьвается с использованием вложения $V_{n}=V_{n, 2} \subset V_{n+1,3}$ и $C$-существенности множества $A(g)$ тем же способом, как в теореме 6 из [14] или в теореме 7 из [4].

Например, докажем утверждение для множества $R_{1}$. Заметим, что $R_{1}$ получается из $R_{0}$ добавлением одной точки - северного полюса.

Положим $q=p^{2}, n=p^{2}-2 p+4$ и пусть $f: S^{n} \rightarrow \mathbb{R}$. Нам будет удобно представлять точку многообразия $V_{n, 2}$ (соответственно $\left.V_{n+1,3}\right)$ как $q$-набор (соответственно $(q+1)$-набор) точек сферы, как описано в конце п. 1 . Например, $x \in V_{n+1,3}$ представляется в виде $x=\left(v_{1}, \ldots, v_{q+1}\right)$, причем множество точек $\left\{v_{1}, \ldots, v_{q+1}\right\}$ получается из $R_{1}$ некоторьм врашением $A \in S O(n+1)$ и элементы группы $G$ переставляют первые $q$ точек в наборе.

Положим

$$
C(f)=\left\{\left(v_{1}, \ldots, v_{q+1}\right) \in V_{n+1,3} \mid f\left(v_{1}\right)=\cdots=f\left(v_{q+1}\right)\right\}
$$

Определим функции $g, h: V_{n+1,3} \rightarrow \mathbb{R}$ формулами

$$
g(x)=f\left(v_{1}\right), \quad h\left(v_{1}, \ldots, v_{q+1}\right)=f\left(v_{q+1}\right)-\max _{1 \leqslant i \leqslant q} f\left(v_{i}\right),
$$

где $x=\left(v_{1}, \ldots, v_{q+1}\right) \in V_{n+1,3}$. Заметим, что функция $h$ непрерьвна и постоянна на орбитах $G$-действия и $C(f)=A(g) \cap h^{-1}(0)$.

Соединим (произвольные) максимум и минимум функции $f$ дугой большого радиуса и параметризуем эту дугу отрезком $I$. Обозначим точки на дуге через $e_{t}, t \in I$, и будем считать, что $e_{0}$ - точка минимума функции $f$, а $e_{1}$ - точка максимума. Как объяснялось в конще п. 1 , имеется эквивариантное вложение $j: V_{n, 2} \times I \subset V_{n+1,3}$, при котором $\nu_{q+1}(j(x, t))=e_{t}$ для любого $x \in V_{n, 2}$, где $\nu_{q+1}: V_{n+1,3} \rightarrow S^{n}$ - проекция на последний вектор, определенная формулой $\nu_{q+1}(x)=v_{q+1}, x=\left(v_{1}, \ldots, v_{q+1}\right)$.

Положим

$$
\bar{g}=g \circ j, \quad \bar{h}=h \circ j: V_{n, 2} \times I \rightarrow \mathbb{R}
$$


Из следствия 1 вытекает, что в $A(\bar{g})$ имеется компонента, соединяющая основания цилиндра $V_{n, 2} \times I$. Кроме того, $\bar{h}$ принимает неположительные значения на нижнем основании цилиндра и неотрицательные на верхнем основании. Из этих двух фактов следует, что $A(\bar{g}) \cap \bar{h}^{-1}(0) \neq \varnothing$; поэтому и $C(f) \neq \varnothing$.

ЗАмЕчаниЕ 5 . При $p=5$, т.е. в случае $\left|R_{0}\right|=25$, из замечания 3 следует, что $k\left(R_{0}\right) \leqslant 16$.

ЗАмЕчАниЕ 6 . Пусть $R_{0}$ - множество вершин правильного 27 -угольника, вписанного в экватор единичной двумерной сферы. Так же, как и выше, по $R_{0}$ определим множество $R_{\rho}, \rho>0$. Тогда из замечания 4 следует, что $k\left(R_{0}\right) \leqslant 20$ и $k\left(R_{\rho}\right) \leqslant 21$.

ЗАмЕЧАнИЕ 7. Используя лемму 1 и теорему 1 , можно получить аналог следствия 1 для случая действия на $V_{n}$ группы $\mathbb{Z}_{p^{\alpha}}, \alpha \geqslant 3$ (в том числе и для отображений в $\mathbb{R}^{m} \mathrm{c}$ $m \geqslant 1)$. Это даст, в частности, как в теореме 3 , оценку сверху на $k\left(R_{0}\right)$ и $k\left(R_{\rho}\right)$, где $R_{0}-$ множество вершин правильного $p^{\alpha}$-угольника, вписанного в экватор единичной двумерной сферы. Однако при $\alpha \geqslant 3$ и $p^{\alpha} \neq 27$ получающаяся оценка, как нетрудно проверить, больше, чем предположенная Кнастером (за неимением лучшей, такая оценка, возможно, представляет некоторьй интерес). Таким образом, предложенный метод приводит к положительному решению лишь в случае групा симметрии $\mathbb{Z}_{p^{2}}(p-$ нечетное простое число) и $\mathbb{Z}_{27}$.

Утверждение теоремы 3 относительно множества $R_{\rho}$ может быть рассмотрено с более общих позиций.

Пусть $R=\left\{v_{1}, \ldots, v_{s}\right\}$ - множество точек на сффере $S^{k-1}$, где $s=|R|$. Обозначим через $l$ размерность линейной оболочки радиус векторов точек из $R$. Множество точек вида $\left(A v_{1}, \ldots, A v_{s}\right)$, где $A$ пробегает $S O(k)$, гомеоморфно многообразию Штифеля $V_{k, l}$ ортонормированных $l$-реперов в $\mathbb{R}^{k}$.

Определим $\nu_{d}: V_{k, l} \rightarrow S^{k-1}$ как проекцию на $d$-й вектор, т.е. формулой $\nu_{d}(P)=A v_{d}$, где $P=\left(A v_{1}, \ldots, A v_{s}\right) \in V_{k, l}, d=1, \ldots, s$.

Для $f: S^{k-1} \rightarrow \mathbb{R}$ положим

$$
B(f)=\left\{x \in V_{k, l} \mid f\left(\nu_{1}(x)\right)=\cdots=f\left(\nu_{s}(x)\right)\right\}
$$

$C$-существенность множества $B(f)$ определяется таким же образом, как и $C$-сушественность $A(f)$, и означает следующее: мы можем определить $B(F)$ для гомотопии $F: S^{k-1} \times I \rightarrow \mathbb{R}$ отображения $f$ формулой

$$
B(F)=\left\{(x, t) \in V_{k, l} \times I \mid f_{t}\left(\nu_{1}(x)\right)=\cdots=f_{t}\left(\nu_{s}(x)\right)\right\}
$$

где $f_{t}(y)=F(y, t), y \in S^{k-1}$. Тогда $B(f)$ называется $C$-сушественным, если для любой гомотопии $F$ отображения $f$ в $B(F)$ имеется компонента связности, соединяющая основания цилиндра.

Скажем, что $R \quad C$-существенно (при данном $k$ ), если $C$-существенным является $B(f)$ при любом $f$.

Например, $C$-существенным является множество $R_{0}$ из теоремы 3 .

По множеству $R \subset S^{k-1}$ построим множество $R_{\rho} \subset S^{k}, \rho>0$, такое, что $\left|R_{\rho}\right|=|R|+1$, следуюшим образом. Будем, как и вьше, считать, что единичная сфера $S^{k-1}$ является 
экватором в $S^{k} \subset \mathbb{R}^{k+1}$. Обозначим через $S_{r}^{k-1}$ сферу радиуса $r<1$, являющуюся пересечением $S^{k}$ с гиперплоскостью, параллельной экваториальной гиперплоскости (таких сфер ровно две). Экватор обозначим через $S_{1}^{k-1}$. Пусть $\rho=h / r$, где $h$ - расстояние меж ду северным полюсом сферы $S^{k}$ и центром сферы $S_{r}^{k-1}$. Тогда $\rho-$ произвольное положительное число. Обозначим через $r R$ множество, полученное из $R$ гомотетией с параметром $r$. Сдвигом перенесем множество $r R$ на сфферу $S_{r}^{k-1}$ и добавим к полученному множеству северньй полюс сферы $S^{k}$. Определенное таким образом множество и есть $R_{\rho}$. В частности, $R_{1}$ получается из $R$ добавлением северного полюса сферы $S^{k}$.

ПРЕДЛОЖЕНИЕ. Если $R \subset S^{k-1}$ является $C$-существенным, то $k\left(R_{\rho}\right) \leqslant k$.

Предложение доказывается таким же образом, как утверждение теоремы 3 , касающееся множества $R_{\rho}$.

Разнообразные применения результатов типа теоремы 3 к задачам комбинаторной геометрии были найдены В. В. Макеевым в [2], [7], [12], [21]. Используя следствие 1, легко получить аналогичные результатам из [2], [7], [12], [21] утверждения о возможности вписать (описать) правильньй $p^{2}$-угольник, имеющий центр в произвольной внутренней точке, в вьпуклое тело размерности $\geqslant p^{2}-2 p+4$ (вокруг двумерного сечения такого выпуклого тела), об $\varepsilon$-асферических двумерных сечениях выпуклых тел и о разрезании конечной массы, непрерывно распределенной в $\mathbb{R}^{n}$, специальным $\mathbb{Z}_{p^{2}}$-симметричным множеством, составленным из $p^{2}$ полугиперплоскостей.

В качестве еще одного геометрического примера покажем, что в компактное выпуклое тело подходящей размерности можно вписать правильную пирамиду, в основании которой лежит правильный $p^{2}$-угольник, гомотетичную вьпуклой оболочке множества $R_{\rho}, \rho>0$. Такую пирамиду будем обзначать через $K_{\rho, r, O}$, где $r$ - радиус описанной двумерной сферы, $O$ - центр этой сферы и $\rho$ - отношение высоты пирамиды к радиусу описанной вокруг основания окружности. Заметим, что две пирамиды с одинаковьми $\rho, r$ и $O$ получаются друг из друга вращением относительно точки $O$.

Из теоремы 3 вытекает следующее утверждение.

СЛЕДСТВИЕ 2. Пусть $V$ - компактное выпуклое тело с непустой внутренностью в $\mathbb{R}^{p^{2}-2 p+5}$. Тогда для любого $\rho>0$ и любой внутренней точки $O \in V$ найдется пирамида типа $K_{\rho, r, O}$, вписанная в $V$.

ДоказАтельство. Рассмотрим единичную сферу с центром в точке $O$ и множество $R_{\rho}$ на ней. Для точки $x$ сферы обозначим через $f(x)$ расстояние между точкой $O$ и гранищей тела $V$ по лучу, соединяющему $O$ с $x$. Таким образом, возникает функция $f$ на $\left(p^{2}-2 p+4\right)$-мерной сфере. Применяя к этой функции теорему 3 , получаем требуемое.

Соответствующие геометрические результаты могут быть получены и с использованием группы симметрии $\mathbb{Z}_{p^{\alpha}}, \alpha>2$ (см. замечание 7 ).

В заключение для удобства читателя приведем вытекающие из [4], [7], [12]-[14] оценки для $k\left(R_{0}\right)$ и $k\left(R_{\rho}\right)$ в случае $\alpha=1$, т.е. когда $\left|R_{0}\right|=p,\left|R_{\rho}\right|=p+1, G=\mathbb{Z}_{p}(p-$ нечетное простое число). Кроме того, приведем аналогичные оценки для множеств $R_{0}^{\prime}$ и $R_{\rho}^{\prime}$, получаюшихся соответственно из $R_{0}$ и $R_{\rho}$ удалением одной из вершин правильного $p$-угольника. Имеем $\left|R_{0}^{\prime}\right|=p-1$ и $\left|R_{\rho}^{\prime}\right|=p$ для $\rho>0$.

Из [7] и [13], [14] следует, что $k\left(R_{0}\right) \leqslant(p+1) / 2$. В [7] показано, что $k\left(R_{0}^{\prime}\right) \leqslant(p-1) / 2$. Так же, как в [4], [14], на основе соображений $C$-существенности доказьвается, что $k\left(R_{\rho}\right) \leqslant(p+3) / 2$ и $k\left(R_{\rho}^{\prime}\right) \leqslant(p+1) / 2$ для $\rho>0$. 
Автор благодарит рецензента за ценные замечания.

\section{СПИСОК ЦИТИРОВАННОЙ ЛИТЕРАТУРЫ}

[1] Knaster B. Problem 4 // Colloq. Math. 1947. №1. P. 30.

[2] Макеев В. В. О некоторых вопросах непрерывных отображений сфер и задачах комбинаторной геометрии // Геометрические вопросы теории функций и множеств. Калинин, 1986. C. $75-85$.

[3] Бабенко И. К., Богатый С. А. К отображению сферы в евклидово пространство // Матем. заметки. 1989. Т. 46. № 3. С. 3-8.

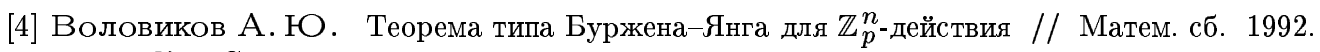
T. 183. № 7. C. 115-144.

[5] Chen W. Counterexamples to Knaster's conjecture // Topology. 1998. V. 37. P. 401-405.

[6] Богатый С. А. Топологические методы в комбинаторных задачах // УМН. 1986. Т. 41. №6. C. $37-47$.

[7] Макеев В. В. Задача Кнастера и почти сферические сечения // Матем. сб. 1989. Т. 180. № 3. C. 424-431.

[8] Floyd E. E. Real-valued mappings of spheres // Proc. Amer. Math. Soc. 1955. V. 6. P. 19-22.

[9] Yamabe H., Yujobô Z. On the continuous functions defined on a sphere // Osaka Math. J. 1950. V. 2. №1. P. 19-22.

[10] Yang C. T. On theorems of Borsuk-Ulam, Kakutani-Yamabe-Yujobô and Dyson, I // Ann. of Math. 1954. V. 60. № 2. P. 262-282.

[11] Munkholm H.J. On the Borsuk-Ulam theorem for $\mathbb{Z}_{p^{\alpha}}$ actions on $S^{2 n-1}$ and maps $S^{2 n-1} \rightarrow \mathbb{R}^{m}$ // Osaka J. Math. 1970. V. 7. P. 451-456.

[12] Макеев В.В. Пространственные обобщения некоторых теорем о выпуклых фигурах // Матем. заметки. 1984. Т. 36. № 3. С. 405-415.

[13] Воловиков А. Ю. К теореме Янга о функциях на сфере // IX Всесоюзная геометрическая конференция. Тез. сообщ. Кишинев: Штиница, 1988. С. 68-69.

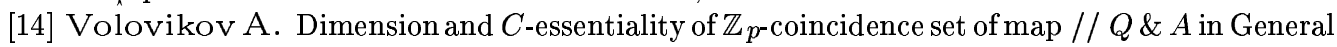
Topology. 1990. V. 8. Spec. Issue. P. 207-217.

[15] Serre J.-P. Homologie singuliére des espaces fibrés. Applications // Ann. Math. 1951. V. 54. P. $425-505$.

[16] Borel A. Sur la cohomologie des espaces fibrés principaux et des espaces homogenes de groupes de Lie compacts // Ann. of Math. (2). 1953. V. 57. P. 115-207.

[17] Шварц А. С. Некоторые оценки рода топологического пространства в смысле Красносельского // УМН. 1957. Т. 12. № 4. С. 209-214.

[18] Munkholm H. J., Nakaoka M. The Borsuk-Ulam theorem and formal group laws // Osaka J. Math. 1972. V. 9. P. 337-349.

[19] Alexander J. C., Yorke J. A. The homotopy continuation method: numerically implementable topological procedures // Trans. Amer. Math. Soc. 1978. V. 242. P. 271-284.

[20] Alligood K.T. Topological conditions for continuation of fixed points // Lecture Notes in Math. 1981. № 886 . P. 20-32.

[21] Макеев В. В. Задача Кнастера о непрерьвньг отображениях сферы в евклидово пространство // Исследования по топологии 6. Зап. науч. семинаров ЛОМИ. Т. 167. Л.: Наука, 1988. C. $169-178$.

Московский государственный институт радиотехники,

Поступило электроники и автоматики

06.04.1999

Исправленный вариант

27.06 .2000 